\title{
Magnetization of $\alpha^{\prime}$ iron nitride produced through the fcc $\rightarrow$ bct martensitic transformation in high magnetic field
}

\author{
T. Koyano ${ }^{\text {a) }}$ and T. Nomiyama \\ Cryogenics Division, Research Facility Center, University of Tsukuba, Tsukuba, Ibaraki 305-8577, Japan \\ N. Kanoh, H. Numata, and T. Ohba \\ Department of Materials Science, Shimane University, Matsue, Shimane 690-8504, Japan \\ E. Kita \\ Institute of Applied Physics, University of Tsukuba, Tsukuba, Ibaraki 305-8573, Japan \\ H. Ohtsuka \\ Tsukuba Magnet Laboratory, National Institute for Materials Science, Tsukuba, Ibaraki 305-0003, Japan
}

(Received 3 March 2006; accepted 22 May 2006; published online 9 August 2006)

\begin{abstract}
The $\gamma$ iron nitride (nitrogen austenite) was subjected to high magnetic field process in order to drive the fcc $\rightarrow$ bct martensitic transformation. Molar fraction of martensite monotonically increased with increasing the magnetic field and reached $94 \%$ at $35 \mathrm{~T}$. With a combination of magnetization and ${ }^{57} \mathrm{Fe}$ Mössbauer spectroscopy data, magnetization of bulk processed $\alpha^{\prime}$ phase with 9.6 at. $\% \mathrm{~N}$ is determined to be $229 \mathrm{emu} / \mathrm{g}$, the same as that for dc sputtered thin films. (C) 2006 American Institute of Physics. [DOI: 10.1063/1.2227640]
\end{abstract}

\section{INTRODUCTION}

The value of spontaneous magnetization is one of fundamental data for ferromagnetic materials. In quest of high performance, a material with magnetization exceeding the $\mathrm{Fe}_{65} \mathrm{CO}_{35}$ alloy has been sought for many years. Kim and Takahashi found that iron films grown in nitrogen gas atmosphere possess larger magnetization than bulk iron and attributed it to the metastable nitride with bct structure, $\alpha^{\prime \prime}$ $-\mathrm{Fe}_{16} \mathrm{~N}_{2}{ }^{1}$. Their discovery generated a huge scientific and technological interest and some research groups successfully reproduced so called the "giant magnetization" of 2.6-3.2 T. ${ }^{2-7}$ But the magnetization of the $\alpha^{\prime \prime}$ phase has been a matter of considerable disagreement among various investigators $^{1-12}$ and discussed in terms of the unit cell volume and degree of order. ${ }^{11,13}$

Another ferromagnetic bct phase, the $\alpha^{\prime}$ phase, exists in the iron-nitrogen system and is often referred as nitrogen martensite. The high temperature fcc phase ( $\gamma$ phase or nitrogen austenite) spontaneously undergoes the fcc $\rightarrow$ bct martensitic transformation when it is quenched below the martensite start temperature $\left(M_{s}\right)$, and the $\alpha^{\prime}$ phase is formed. Because nitrogen atoms in $\gamma$ phase are irregularly distributed on the octahedral interstitial sites in the fcc lattice of iron and this transformation proceeds without diffusion process, nitrogen atoms are arranged in a disordered manner on octahedral sites along the $c$ axis in the $\alpha^{\prime}$ phase. ${ }^{14}$ Heat treatment around $393 \mathrm{~K}$ orders nitrogen atoms, and the $\alpha^{\prime \prime}$ phase is formed. ${ }^{15}$ It can be said that the $\alpha^{\prime}$ phase is a precursor to obtain the $\alpha^{\prime \prime}$ phase. Because the martensitic transformation accompanies a large atomic volume increase, the strain energy is accumulated in the matrix as the structure change proceeds. This tends to suppress further progress of the transformation. As a result, the $\alpha^{\prime}$ phase always coexists with the

\footnotetext{
${ }^{a)}$ Electronic mail: koyano@bk.tsukuba.ac.jp
}

retained austenite in a sample as far as high nitrogen $\gamma$ phase is concerned. Therefore, precise molar fraction of the $\alpha^{\prime}$ phase needs to be obtained by some means when one would like to determine its intrinsic magnetization with magnetic measurements. Huang et al. produced fine powders consisted of $56 \mathrm{wt} \% \alpha^{\prime}$ phase and estimated that the magnetization is $250 \mathrm{emu} / \mathrm{g}$ by an extrapolation of $\mathrm{x}$-ray diffraction intensities. $^{4-6}$ Using thin film technique, single phase samples of the $\alpha^{\prime}$ phase can be obtained and, therefore, the error associated with the molar fraction estimation can be avoided. But the reported values tend to be scattered ranging from 228 to $257 \mathrm{emu} / \mathrm{g} .{ }^{3,8,9,11}$ Therefore, it is necessary to determine the intrinsic value using substrate-free bulk samples of high molar fraction. Having the reliable value for the $\alpha^{\prime}$ phase, magnetization change upon annealing would provide valuable information whether the giant magnetization exists or not in the $\alpha^{\prime \prime}$ phase.

We have applied high magnetic fields up to $40 \mathrm{~T}$ to the $\gamma$ phase foils at low temperatures in order to increase the molar fraction of the $\alpha^{\prime}$ phase. ${ }^{16-18}$ Magnetic field lowers the Gibbs free energy of ferromagnetic martensite phase whereas no substantial change takes place for the paramagnetic parent phase. ${ }^{19}$ As a result, the $\gamma \rightarrow \alpha^{\prime}$ martensitic transformation is promoted. Therefore, molar fraction of the $\alpha^{\prime}$ phase can be changed with the intensity of magnetic field, and a higher fraction can be achieved than by just cooling the $\gamma$ phase to the helium temperature in zero magnetic field. Taking full advantage of the magnetic field effect, the intrinsic magnetization of the $\alpha^{\prime}$ phase can be determined by measuring magnetization of samples as a function of the molar fraction and extrapolation to $100 \%$. The molar fraction exceeding $90 \%$ of high magnetic field samples ${ }^{18}$ enables high precision determination of the intrinsic value.

In this report, precise magnetic measurements and ${ }^{57} \mathrm{Fe}$ transmission Mössbauer spectroscopy were carried out in or- 
der to determine the intrinsic magnetization of bulk processed $\alpha^{\prime}$ phase. And we propose another method to reduce the retained austenite having a potentiality to produce a large amount of the $\alpha^{\prime}$ phase.

\section{EXPERIMENTAL PROCEDURE}

Iron foils (99.9\% in purity) were annealed at $1270 \mathrm{~K}$ in dry hydrogen gas in order for grain growth for $1 \mathrm{~h}$ and successively nitrified in a mixture of $\mathrm{NH}_{3}$ and $\mathrm{H}_{2}$ gas at $923 \mathrm{~K}$ for $1 \mathrm{~h}$. Their thickness was $0.01 \mathrm{~mm}$ to minimize inhomogeneity of nitrogen concentration. Samples were quenched directly from the nitriding temperature to distilled water to avoid decomposition into a mixture of $\alpha$-Fe and $\gamma^{\prime}-\mathrm{Fe}_{4} \mathrm{~N}$. All samples were checked with $\mathrm{x}$-ray diffraction using Mo $K \alpha$ radiation and are found to be a single phase of $\gamma$. The Nelson-Riley extrapolation ${ }^{20}$ with 23 diffraction lines was used to obtain lattice parameters of the best possible accuracy after deconvolution of $K \alpha_{1}$ and $K \alpha_{2}$ lines. Samples with lattice parameter of $3.646 \AA \leqq a_{0} \leqq 3.647 \AA$ were selected for the following experiments. Corresponding nitrogen concentration was 9.6 at. $\%{ }^{21}$ The surface of nitrified foils was shiny silver and no surface treatment was applied during the course of the present experiment. The $\gamma$ phase samples were immersed into liquid helium in zero magnetic field, and then external magnetic field was applied. A conventional superconducting magnet and a Bitter-type magnet were used to generate magnetic field of $0 \mathrm{~T}<B \leqq 5 \mathrm{~T}$ and $10 \mathrm{~T} \leqq B$ $\leqq 23 \mathrm{~T}$, respectively. For experiments up to $35 \mathrm{~T}$, a hybrid magnet consisting of $14 \mathrm{~T}$ superconducting and $21 \mathrm{~T}$ Bittertype magnets was employed. The field was applied in the sample plane to minimize the demagnetization factor. After decreasing the field to zero, samples were heated up to room temperature and $\mathrm{x}$-ray diffraction, magnetic measurements, and ${ }^{57} \mathrm{Fe}$ Mössbauer spectroscopy were carried out.

Magnetization curves were measured at $280 \mathrm{~K}$ using a superconducting quantum interference device (SQUID) magnetometer (Quantum Design MPMS-5) in magnetic field of $-4 \mathrm{~T} \leqq B \leqq 4 \mathrm{~T}$. Magnetic field was applied in the sample plane. In order to obtain a precise determination of magnetization, all samples were trimmed into $5 \times 10 \mathrm{~mm}^{2}$ in size after the high field process at $4.2 \mathrm{~K}$, and then magnetization was measured. The magnetization scale of the magnetometer was calibrated with regard to the sample size $\mathrm{e}^{22}$ assuming the spontaneous magnetization of pure iron as $218 \mathrm{emu} / \mathrm{g}$ at $293 \mathrm{~K}^{23}$ The samples taken out from the magnetometer were successively used for ${ }^{57} \mathrm{Fe}$ Mössbauer spectroscopy. The absorption spectra were recorded at room temperature using a spectrometer calibrated with a reference to pure $\alpha$-Fe.

\section{RESULTS AND DISCUSSION}

The $\gamma$ phase of present nitrogen concentration possesses the martensite start temperature $\left(M_{s}\right)$ of about $200 \mathrm{~K}$ in zero magnetic field ${ }^{24}$ and starts to transform into the $\alpha^{\prime}$ phase when it is cooled to that temperature. Since Gibbs free energy for the $\alpha^{\prime}$ phase is lower than that of $\gamma$ phase all over the temperature range studied, the $\alpha^{\prime}$ phase never returns to

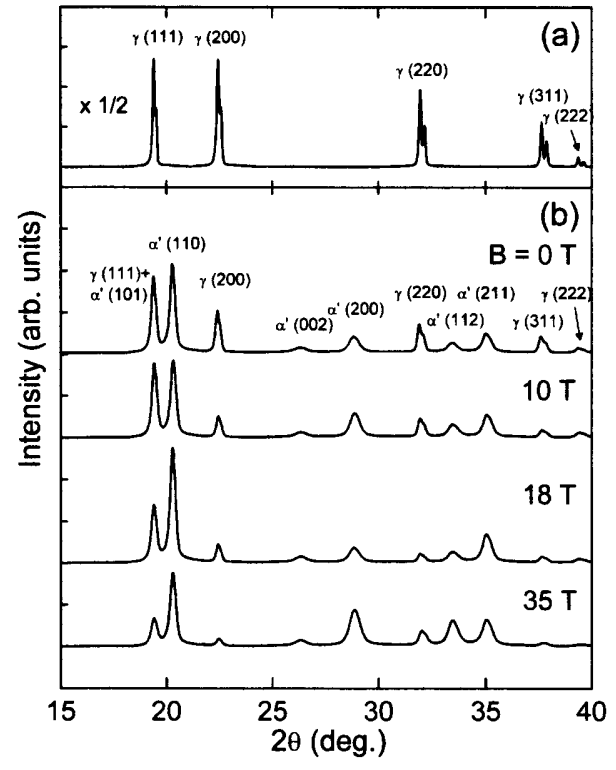

FIG. 1. X-ray diffraction spectra for as-quenched $\gamma$ phase (a) and $\gamma$ phase samples cooled to $4.2 \mathrm{~K}$ and subjected to various magnetic fields (b). Incident $\mathrm{x}$-ray intensity and irradiated sample area were the same for all spectra. Measurements were done at room temperature using Mo $K \alpha$ radiation.

the $\gamma$ phase if it were once formed. Therefore, the molar fraction of $\gamma$ phase is preserved upon heating to room temperature.

$\mathrm{X}$-ray diffraction spectra for as-quenched $\gamma$ phase and the samples subsequently cooled to $4.2 \mathrm{~K}$ are plotted in Fig. 1 as a function of applied magnetic field at that temperature. The $\gamma$ phase foils are polycrystalline and all diffraction lines are indexed in terms of single fcc structure. Diffraction lines associated with the $\alpha^{\prime}$ phase emerged by cooling the $\gamma$ phase to $4.2 \mathrm{~K}$. Relative diffraction intensity of the $\gamma$ phase decreases with increasing the applied magnetic field as can be seen from the $\gamma(200)$ and (220) lines. This indicates that magnetic field drives the $\gamma \rightarrow \alpha^{\prime}$ martensitic transformation as reported earlier. ${ }^{16-18}$ The $\gamma$ phase lines are still observable with application of $35 \mathrm{~T}$, and a higher magnetic field is found to be needed to obtain single phase of $\alpha^{\prime}$. As opposed to the $\gamma$ phase lines, the magnetic field dependence of line intensity is complicated for the $\alpha^{\prime}$ phase. In fact, the intensity of $\alpha^{\prime}(002)$ line is the same regardless of the applied magnetic field, but other lines show apparent field dependence. In present measurements, the scattering vector is normal to the foil surface and magnetic field was applied in the plane. The $\alpha^{\prime}$ phase is reported to possess a large uniaxial magnetocrystalline anisotropy of $4 \times 10^{6} \mathrm{erg} / \mathrm{cm}^{3}$ along the $c$ axis. $^{25}$ Therefore, the free energy of the $\alpha^{\prime}$ phase variants with $c$ axis parallel to magnetic field is lower than those of different orientations. If we supposed that the $\alpha^{\prime}$ phase variants having its $c$ axis in the direction of magnetic field preferentially nucleated when magnetic field was applied, the volume of variants with the $c$ axis perpendicular to the foil surface would not be affected by the presence of magnetic field and would be the same as $0 \mathrm{~T}$ sample regardless of the field intensity. Then, the diffraction intensity of $\alpha^{\prime}(002)$ line is independent of magnetic field and that for the other lines is to be dependent. The volume fraction of the $\alpha^{\prime}$ phase mono- 


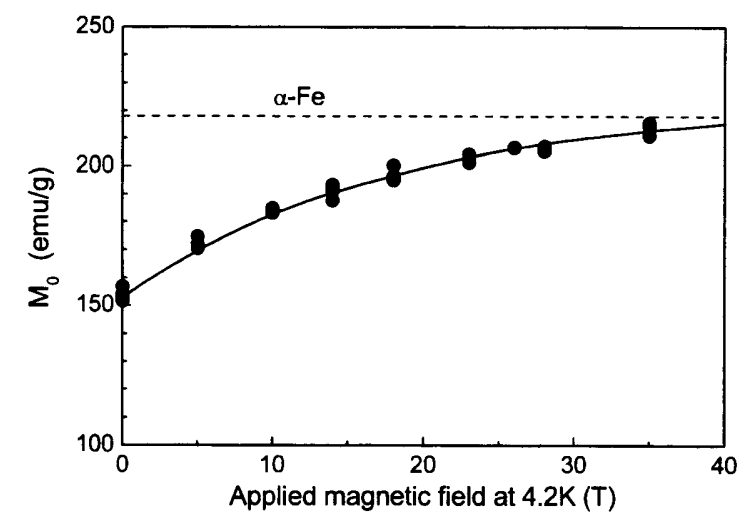

FIG. 2. Spontaneous magnetization at $280 \mathrm{~K}, M_{0}$, for the $\alpha^{\prime}+\gamma$ phase samples as a function of applied magnetic field at $4.2 \mathrm{~K}$.

tonically increases with increasing the magnetic field as can be seen from the intensities of $\gamma$ phase lines, but the diffraction intensities of $\alpha^{\prime}$ phase lines with indices other than (002) do not necessarily increase monotonically. Since variant nucleation process is a statistical plus correlative phenomenon, crystallographic orientation of variants can be somewhat different for sample by sample except for the $\alpha^{\prime}(002)$ line. If this simple explanation successfully described what took place in present samples, it would be possible to produce hardened nitrogen steels comprising $c$-axis-oriented variants by application of magnetic field.

Due to a sharp contrast in magnetic character of the phases involved, progress of the transformation can be monitored quantitatively with magnetic measurements. Spontaneous magnetization of the $\gamma+\alpha^{\prime}$ samples (hereafter abbreviated as $M_{0}$ ) at $280 \mathrm{~K}$ is plotted in Fig. 2 as a function of applied magnetic field at $4.2 \mathrm{~K}$. Here, we define the spontaneous magnetization for each sample as a linear extrapolation of respective magnetization curves in 2.2-4 $\mathrm{T}$ to zero magnetic field. With this calculation, any contribution from paramagnetic $\gamma$ phase to the sample magnetization is completely eliminated. Therefore, the value of $M_{0}$ equals the molar fraction of the $\alpha^{\prime}$ phase, $f$, multiplied by its spontaneous magnetization, $M \alpha^{\prime}$. With increasing the applied magnetic field at $4.2 \mathrm{~K}$, the value of $M_{0}$ monotonically and gradually increases in good accordance with x-ray diffraction data in Fig. 1. The largest $M_{0}$ of $215 \mathrm{emu} / \mathrm{g}$ was observed for $35 \mathrm{~T}$ samples in spite of the presence of $\gamma$ phase. The spontaneous magnetization of pure $\mathrm{Fe}$ is also indicated in the figure as a dotted line for a reference. It seems possible to exceed the value for pure $\mathrm{Fe}$ if far stronger magnetic fields than $35 \mathrm{~T}$ were employed. Pulsed magnets are able to generate higher intensity of magnetic field, but it has been shown to be less effective than static field. ${ }^{16,17}$ Practically, a method should be exploited and needs to be combined with to supplement the magnetic field effect.

In principle, the value of $M \alpha^{\prime}$ can be determined if a set of data, $M_{0}$ and $f$, were obtained. The authors would like to stress that both $M_{0}$ and $f$ should be precisely determined using the same sample for this particular purpose, and great care should to be expended in determining the $f$. Present $\mathrm{x}$-ray diffraction data would offer valuable information concerning the molar fraction of phases involved but the pre-

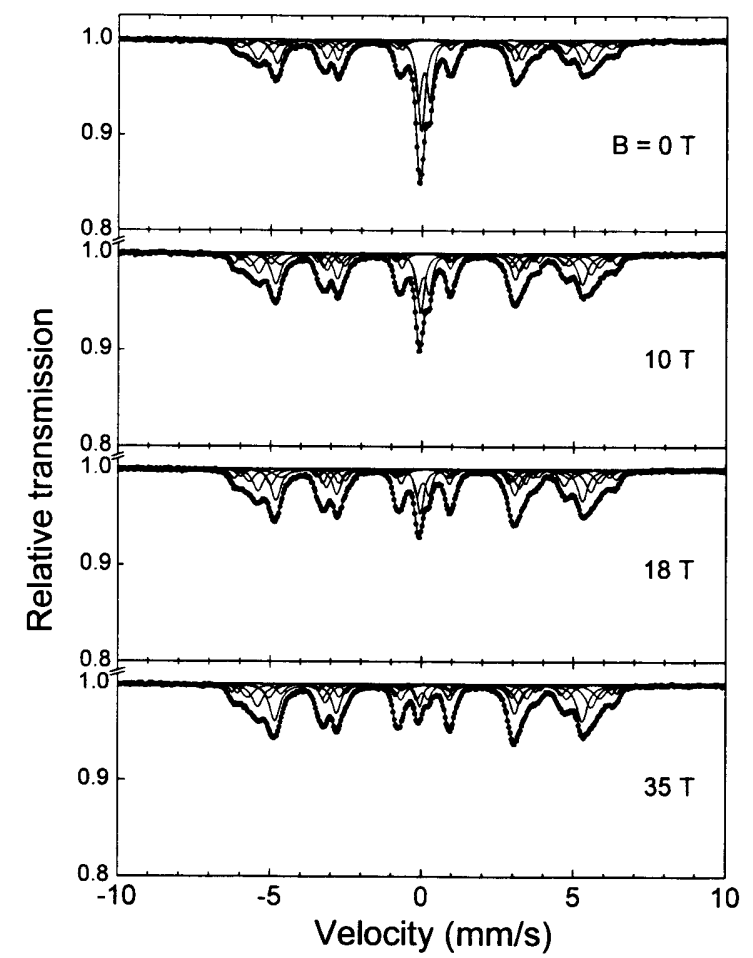

FIG. 3. ${ }^{57} \mathrm{Fe}$ Mössbauer spectra for the $\alpha^{\prime}+\gamma$ phase samples subjected to various magnetic fields at $4.2 \mathrm{~K}$. All spectra were measured at room temperature.

ferred orientation of crystallites hampers the precise determination. The samples can be powdered in order to remove the textured structure, but the fraction would become different from the value before milling instead because of the deformation induced martensitic transformation. Therefore, we have selected ${ }^{57} \mathrm{Fe}$ Mössbauer spectroscopy in the transmission geometry which is capable of measuring the samples as taken out from the SQUID magnetometer. Figure 3 shows Mössbauer spectra for the $\alpha^{\prime}+\gamma$ phase samples for various applied magnetic fields at $4.2 \mathrm{~K}$. A prominent paramagnetic absorption of the $\gamma$ phase is observed around zero velocity besides a convolution of magnetic sextets originating in the $\alpha^{\prime}$ phase. As can be seen in the figure, the $\gamma$ phase absorption becomes weaker with increasing the magnetic field in good accordance with Figs. 1 and 2 . The spectra were analyzed in order to determine the absorption area ratio of the $\alpha^{\prime}$ and $\gamma$ phases using a least squares fitting software with an assumption of Lorentzian line shape and the thin absorber approximation. Oda et al. ${ }^{26}$ and Hinomura and $\mathrm{Nasu}^{27}$ reported that the $\gamma$ phase spectrum needs to be analyzed with a singlet plus two sets of doublet. Since the absorption area ratio of the second doublet is only $3.4 \%$ of the total $\gamma$ phase absorption, ${ }^{27}$ we have analyzed the central paramagnetic absorption with a combination of a singlet and a symmetric doublet for simplicity. The absorption spectrum for the $\alpha^{\prime}$ phase is composed of many sextets and characterized with a broad linewidth as deduced from its disordered structure. In order for the precise absorption area calculation of the $\alpha^{\prime}$ phase, seven sets of sextet with the first order (magnetic +quadrupole) interaction were found to be needed. Absorption area ratio for individual lines in each sextet was assumed to be $3: x: 1: 1: x: 3$, where the $x$ is a free parameter to 
TABLE I. Hyperfine parameters and absorption area ratio for the $35 \mathrm{~T}$ sample at room temperature.

\begin{tabular}{ccccc}
\hline \hline Subspectum & $\begin{array}{c}\text { Hhf } \\
(\mathrm{kOe})\end{array}$ & $\begin{array}{c}\text { IS } \\
(\mathrm{mm} / \mathrm{s})\end{array}$ & $\begin{array}{c}\text { QS } \\
(\mathrm{mm} / \mathrm{s})\end{array}$ & $\begin{array}{c}\text { Area ratio } \\
(\%)\end{array}$ \\
\hline$\gamma^{1}$ & 0 & -0.11 & 0.00 & 2.8 \\
$\gamma^{2}$ & 0 & 0.03 & 0.39 & 3.7 \\
$\alpha^{\prime 1}$ & 314 & 0.18 & 0.10 & 30.6 \\
$\alpha^{\prime 2}$ & 341 & 0.05 & 0.08 & 20.9 \\
$\alpha^{\prime 3}$ & 360 & 0.08 & -0.03 & 13.7 \\
$\alpha^{\prime 4}$ & 304 & 0.00 & -0.26 & 12.1 \\
$\alpha^{\prime 5}$ & 286 & 0.09 & -0.23 & 7.3 \\
$\alpha^{\prime 6}$ & 382 & 0.13 & -0.09 & 5.2 \\
$\alpha^{\prime 7}$ & 393 & 0.16 & -0.14 & 3.7 \\
\multicolumn{7}{c}{ Normalized chi-square } & & & 1.68 \\
\hline \hline
\end{tabular}

allow any appreciable magnetic anisotropy effect, and all sextets were constrained to possess the same value of $x$. With this model, all spectra were successfully analyzed, and the hyperfine parameters and absorption area ratio of respective components for the $35 \mathrm{~T}$ spectrum are summarized in Table I. No substantial difference in the hyperfine parameters was observed for samples of various magnetic fields, whereas the field dependence of the absorption area ratio for the $\gamma$ phase, $A \gamma$, was prominent. The value of $x$ was found to be 1.932.22 .

The values of $f$ and $M \alpha^{\prime}$ can be directly derived from a single set of $A \gamma$ and $M_{0}$ if we assumed the same recoil-less fraction for the $\alpha^{\prime}$ and $\gamma$ phases and ignored the finite absorber thickness effect. ${ }^{26,28}$ If these primitive calculations were applicable, the value of $M_{0}$ should be exactly proportional to $A \gamma$, and all data points fall on a straight line connecting two points on the horizontal and vertical axes $(100$, $0)$ and $\left(0, M \alpha^{\prime}\right)$, respectively, in a diagram where $M_{0}$ were plotted against $A \gamma$. Deviation from the straight line should be attributed to either or both the finite absorber thickness effect and/or differences in the recoil-less fraction. The value of $M_{0}$ is plotted as a function of $A \gamma$ in Fig. 4 and seems to decrease linearly with increasing the $A \gamma$. Apparently, data points locate below the straight line and show larger gradient. Therefore, the $M \alpha^{\prime}$ cannot be determined with a single set of $A \gamma$

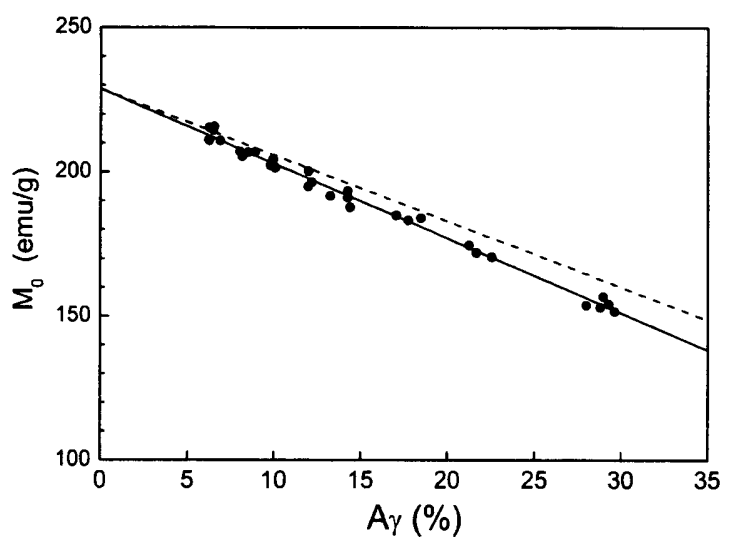

FIG. 4. Spontaneous magnetization at $280 \mathrm{~K}, M_{0}$, of the $\alpha^{\prime}+\gamma$ phase samples plotted against the absorption area ratio of the $\gamma$ phase, $A \gamma$. Dotted straight line connecting $(100,0)$ and $(0,229)$ is drawn to guide the eyes.

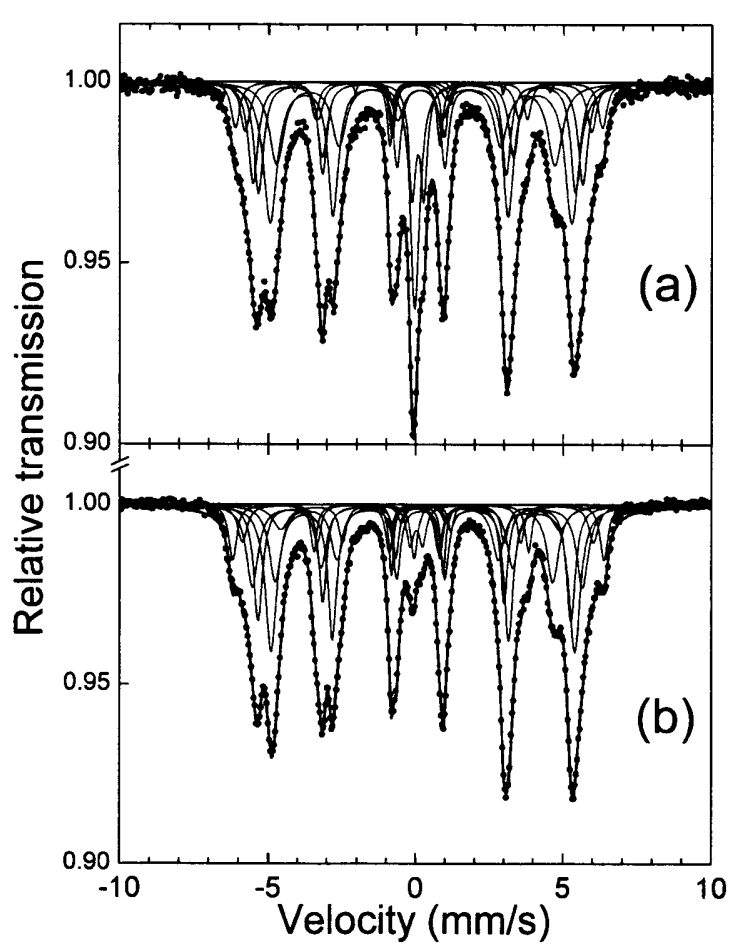

FIG. 5. ${ }^{57}$ Fe Mössbauer spectra for samples subjected to mechanical milling process. Starting materials are the $\gamma$ phase foils for (a) and the $35 \mathrm{~T}$ sample for (b), respectively. The spectra were recorded at room temperature.

and $M_{0}$, and sets of data for samples with different $f$ and the same nitrogen concentration are necessary in order to remove the effects described above. We approximated the data points to a linear function and extrapolated to $A \gamma=0$ for the accurate determination of $M \alpha^{\prime}$. The value of $M \alpha^{\prime}$ is determined to be $229 \mathrm{emu} / \mathrm{g}$. With this value, the highest molar fraction attained was found to be $94 \%$ at $35 \mathrm{~T}$.

Takahashi and Shoji discussed the magnetization of bct iron nitrides as a function of the volume per unit cell. ${ }^{11}$ The lattice parameters of $\alpha^{\prime}$ phase with present nitrogen concentration were determined to be $a=2.8473 \AA$ and $c$ $=3.1113 \AA$ with the Rietvelt analysis of $\mathrm{x}$-ray diffraction data collected at SPring-8 synchrotron facility. ${ }^{29}$ Corresponding unit cell volume is $25.22 \AA^{3}$. Present magnetization and unit cell volume are in good agreement with those for dc sputtered thin films of the $\alpha^{\prime}$ single phase, namely, $228 \mathrm{emu} / \mathrm{g}$ at $25.5 \AA^{3}{ }^{3,11}$ In other words, a high magnetization material exceeding pure iron can be produced not only by the use of thin film technique but also with conventional bulk process when the retained austenite were completely removed. But the value is not as large as $250 \mathrm{emu} / \mathrm{g}$ or $2.4 \mathrm{~T}^{3,5}$

Keeping in mind that plastic deformation of the $\gamma$ phase may result in formation of the $\alpha^{\prime}$ phase even at room temperature through the deformation induced martensitic transformation, we have powdered the $\gamma$ phase foils using a hand mortar in air. Here, iron foils of smaller grain size (without the $1270 \mathrm{~K}$ hydrogen gas treatment) were employed. The powder was initially paramagnetic and turned into ferromagnetic with increasing the grinding time, indicating the progress of the transformation. Figure 5(a) is the Mössbauer spectrum of the powdered $\gamma$ phase sample. The spectrum 
consists of the $\gamma$ and $\alpha^{\prime}$ phase absorption and analyzed with the same procedure as those for high magnetic field samples in Fig. 3. The value of $A \gamma$ is $12.6 \%$ and is almost the same as that for $18 \mathrm{~T}$ sample. Milling of the $\gamma$ phase is found to be capable of producing the $\alpha^{\prime}$ phase without cooling to subzero temperatures or using high magnetic fields. In order to check the effect of grinding after the high magnetic field process, the $35 \mathrm{~T}$ samples were crushed and powdered likewise. The value of $A \gamma$ was reduced to $3.8 \%$ [Fig. 5(b)], which corresponds to the $M_{0}$ of $220 \mathrm{emu} / \mathrm{g}$ in Fig. 4. Present results suggest that milling of the $\gamma$ phase has the potentiality to produce a large amount of bct iron nitride powders with high magnetization.

\section{CONCLUSION}

The magnetization of bulk processed $\alpha^{\prime}$ phase with 9.6 at. $\% \mathrm{~N}$ is determined to be $229 \mathrm{emu} / \mathrm{g}$, the same as that produced by dc sputtering technique. The materials having a larger magnetization than pure iron can be synthesized when the retained austenites were removed. Both high magnetic field process at low temperatures and mechanical milling treatment are effective for this purpose.

\section{ACKNOWLEDGMENTS}

High magnetic field experiments using the hybrid and Bitter-type magnets were carried out at the Tsukuba Magnet Laboratory, National Institute for Materials Science. All measurements and sample preparation were done at Research Facility Center, University of Tsukuba. One of the authors (T.K.) would like to appreciate financial support of Grant-inAid for Scientific Research (C) (2) (12650655) from the Ministry of Education, Culture, Sports, Science and Technology of Japan, The Kurata Research grant, University of Tsukuba Research Initiative, and The Iron and Steel Institute of Japan.
${ }^{1}$ T. K. Kim and M. Takahashi, Appl. Phys. Lett. 20, 492 (1972).

${ }^{2}$ M. Komuro, Y. Kozono, M. Hanazono, and Y. Sugita, J. Appl. Phys. 67, 5126 (1990)

${ }^{3}$ Y. Sugita, H. Takahashi, M. Komuro, K. Mitsuoka, and A. Sakuma, J. Appl. Phys. 76, 6637 (1994).

${ }^{4}$ M. Q. Huang, W. E. Wallace, S. Shimizu, and S. G. Sanker, J. Magn. Magn. Mater. 135, 226 (1994).

${ }^{5}$ M. Q. Huang, W. E. Wallace, S. Shimizu, A. T. Pedziwiatr, R. T. Obermyer, and S. G. Sanker, J. Appl. Phys. 75, 6574 (1994).

${ }^{6}$ W. E. Wallace and H. Q. Huang, J. Appl. Phys. 76, 6648 (1994).

${ }^{7}$ S. Okamoto, S. Kitakami, and Y. Shimada, J. Appl. Phys. 85, 4952 (1999).

${ }^{8} \mathrm{~K}$. Nakajima and S. Okamoto, Appl. Phys. Lett. 56, 92 (1990).

${ }^{9}$ M. Takahashi, H. Shoji, H. Takahashi, H. Nashi, T. Wakiyama, M. Doi, and M. Matsui, J. Appl. Phys. 76, 6642 (1994).

${ }^{10} J$. M. D. Coey, K. O'Donnell, Q. Qinian, E. Touchais, and K. H. Jack, J. Phys.: Condens. Matter 6, L23 (1994).

${ }^{11} M$. Takahashi and H. Shoji, J. Magn. Magn. Mater. 208, 145 (2000).

${ }^{12}$ T. Hattori, N. Kamiya, and Y. Kato, J. Magn. Soc. Jpn. 25, 927 (2001).

${ }^{13}$ S. Okamoto, O. Kitakami, and Y. Shimada, J. Magn. Magn. Mater. 208, $102(2000)$.

${ }^{14}$ K. H. Jack, Proc. R. Soc. London, Ser. A 208, 200 (1951).

${ }^{15}$ K. H. Jack, Proc. R. Soc. London, Ser. A 208, 216 (1951).

${ }^{16}$ T. Koyano, H. Ikeda, R. Yoshizaki, A. Tasaki, T. Takamasu. H. Ohtsuka, $\mathrm{H}$. Wada, and G. Kido, in Proceedings of the International Conference on Solid-Solid Phase Transformations '99 (JIMIC-3), edited by M. Koiwa, K. Ohtsuka, and T. Miyazaki (The Japan Institute of Metals, Sendai, Japan, 1999), pp. 365-368.

${ }^{17}$ T. Koyano et al., Mater. Trans., JIM 41, 923 (2000).

${ }^{18}$ T. Koyano, T. Nomiyama, H. Ikeda, N. Kanoh, T. Ohba, H. Ohtsuka, and H. Wada, Trans. Mater. Res. Soc. Jpn. 28, 235 (2003).

${ }^{19}$ T. Kakeshita, T. Saburi, and K. Shimizu, Mater. Sci. Eng., A 273-275, 21 (1999).

${ }^{20} \mathrm{~J}$. B. Nelson and D. P. Riley, Proc. Phys. Soc. London 57, 160 (1945).

${ }^{21}$ H. A. Wriedt, N. A. Gokcen, and R. H. Nafziger, in Phase Diagrams of Binary Iron Alloys, edited by H. Okamoto (ASM International, Materials Park, OH, 1993), pp. 222-242.

${ }^{22}$ T. Koyano, Jpn. J. Appl. Phys., Part 1 43, 7322 (2004).

${ }^{23}$ R. M. Bozorth, Ferromagnetism (Van Nostrand, New York, 1951), p. 867. ${ }^{24}$ T. Bell, J. Iron Steel Inst., London 206, 1017 (1968).

${ }^{25}$ M. Takahashi, Y. Takahashi, K. Sunaga, and H. Shoji, J. Magn. Magn. Mater. 239, 479 (2002).

${ }^{26}$ K. Oda, K. Umezu, and H. Ino, J. Phys.: Condens. Matter 2, 10147 (1990).

${ }^{27}$ T. Hinomura and S. Nasu, Mater. Trans., JIM 39, 700 (1998).

${ }^{28}$ N. N. Greenwood and T. C. Gibb, Moessbauer Spectroscopy (Chapman and Hall, London, 1971), pp. 30-35.

${ }^{29}$ T. Ohba, N. Kanoh, T. Koyano, H. Ikeda, H. Ohtsuka, H. Wada, and K. Kato, Mater. Trans., JIM 44, 2537 (2003). 